\title{
The Fish Patty Experiment: A Strip-Plot Look
}

\author{
Peter Goos \\ Department of Biosystems, KU Leuven \\ Department of Engineering Management, University of Antwerp
}

July 6,2021

\begin{abstract}
In this article, I provide a detailed discussion of the well-known fish patty experiment introduced in the literature by Cornell (1981). Cornell (1988) used that experiment as the motivating example for an article discussing that, for logistical reasons, many mixture-process variable experiments are run using a split-plot experimental design. More specifically, he described two possible ways in which the fish patty experiment might have been performed, both of which require a split-plot analysis of the data. These descriptions were not followed by the corresponding analyses of the fish patty data. Moreover, Cornell (1988) did not discuss the most convenient way in which the fish patty experiment could have been run, namely using a strip-plot design. In this article, I discuss the logistics leading to a strip-plot design, conduct the corresponding strip-plot analysis and contrast it with the two split-plot analyses.
\end{abstract}

Keywords: completely randomized design, mixture-process variable experiment, split-plot design, strip-plot design 


\section{Introduction}

Mixture experiments are often complicated due to the study of process variables, in addition to the proportions of the mixture ingredients. Cornell (1988) defined process variables as experimental factors that do not form any portion of the mixture but whose levels, when changed, could affect the blending properties of the mixture ingredients. Experimental designs for studying the joint effects of mixture ingredients and process variables are termed mixture-process variable (MPV) designs. Standard models for data from MPV designs have been described in detail in Cornell (2002) and Smith (2005). More parsimonious models have been proposed by Kowalski et al. (2000, 2002). Standard designs for MPV experiments have been described in Cornell (2002). Smaller experimental designs were presented by Cornell and Gorman (1984), Kowalski et al. (2000) and Goos and Donev (2007).

MPV experiments have many applications. For instance, Næs et al. (1998) discussed the analysis of data from an experiment involving a threeingredient mixture and two process variables. The three mixture ingredients were three kinds of wheat, and the two process variables were mixing time and proofing time of a dough. Cho et al. (2012) discussed a MPV experiment to study a diamond grinding wheel. The experiment involved four mixture ingredients and four process variables. Christaki et al. (2017) designed and analyzed an experiment involving five mixture ingredients (two kinds of flour and three kinds of leavening acid) and one process variable (mixing time), to quantify their influence on cake quality. Finally, Akkermans et al. (2017) discussed the design and analysis of excipient compatibility studies. Their example is an experiment in which the joint impact of seven excipients (three fillers, a glidant, a desintegrant, a surfactant and a lubricant) and two process variables (temperature and relative humidity) on the degradation of a drug are studied.

Cornell (1988) pointed out that the combination of mixture ingredients and process variables in a study increases the size of the experimental design. This often renders complete randomization infeasible and necessitates the tests to be grouped somehow. To make his point, Cornell (1988) revisits the fish patty experiment described in Cornell (1981) and Cornell and Gorman (1984). In that experiment, seven mixtures of three fish species (mullet, sheepshead and croaker) were subjected to different processing conditions. There were eight processing conditions, defined by an oven cooking temperature (375 or 425 degrees Fahrenheit), an oven cooking time (25 or 
40 minutes) and a deep fat frying time (25 or 40 seconds). Consequently, there were $7 \times 8=56$ combinations of blends and processing conditions. The design used for the mixture was a 7-point simplex-centroid design (Cornell, $2002)$, while that for the processing conditions was a $2^{3}$ factorial design. The response was the firmness of the patties. More details concerning the practicalities of the fish patty experiment can be found in Chapter 7 of Cornell (2002). The data are shown in Table 1 where the observed firmness is denoted by $y$, the proportions of mullet, sheepshead and croaker are denoted by $x_{1}, x_{2}$ and $x_{3}$, respectively, and the oven cooking temperature, oven cooking time, and deep fat frying time are denoted by $z_{1}, z_{2}$ and $z_{3}$, respectively.

Section 2 lists the steps required to run the fish patty experiment in a completely randomized fashion. Sections 3 and 4 present the split-plot analyses Cornell (1988) discussed for the data from the fish patty experiment. Section 5 compares the results from the two split-plot analyses. Section 6 presents an alternative analysis for the data, assuming that the fish patty experiment was performed using a strip-plot experimental design. Finally, Section 7 summarizes the results.

All analyses reported in this article used a significance level of 0.01 and coded levels of the process variables $(-1$ for their low levels and +1 for their high levels). The final models were obtained using a backward model selection approach. The initial model used for each analysis was a secondorder MPV model recommended by Kowalski et al. (2000) for data from MPV experiments. More specifically, I started from the model

$$
\sum_{i=1}^{3} \beta_{i}^{\mathrm{m}} x_{i}+\sum_{i=1}^{2} \sum_{j=i+1}^{3} \beta_{i j}^{\mathrm{m}} x_{i} x_{j}+\sum_{i=1}^{3} \sum_{j=i+1}^{3} \beta_{i j}^{\mathrm{mp}} x_{i} z_{j}+\sum_{i=1}^{2} \sum_{j=i+1}^{3} \beta_{i j}^{\mathrm{p}} z_{i} z_{j}
$$

for the firmness, where the superscripts ' $m$ ' and 'p' refer to the mixture ingredients and the process variables, respectively.

To decide on the importance of the interaction between the mixture ingredients and a given process variable $z_{j}$, I tested whether or not $\beta_{1 j}^{\mathrm{mp}}=$ $\beta_{2 j}^{\mathrm{mp}}=\beta_{3 j}^{\mathrm{mp}}$. If yes, this implies that the effect of the $j$ th process variable does not depend on the composition of the mixture and I replaced $\beta_{1 j}^{\mathrm{mp}} x_{1} z_{j}+\beta_{2 j}^{\mathrm{mp}} x_{2} z_{j}+\beta_{3 j}^{\mathrm{mp}} x_{3} z_{j}$ with $\beta_{j}^{p} z_{j}$ and tested whether $\beta_{j}^{p}=0$. If no, I left the three terms $\beta_{1 j}^{\mathrm{mp}} x_{1} z_{j}, \beta_{2 j}^{\mathrm{mp}} x_{2} z_{j}$ and $\beta_{3 j}^{\mathrm{mp}} x_{3} z_{j}$ in the model, regardless of the individual $p$ values for $\beta_{1 j}^{\mathrm{mp}}, \beta_{2 j}^{\mathrm{mp}}$ and $\beta_{3 j}^{\mathrm{mp}}$. As it is not interesting to test whether $\beta_{1}^{\mathrm{m}}, \beta_{2}^{\mathrm{m}}$ and $\beta_{3}^{\mathrm{m}}$ are zero, no $t$ ratios or $p$ values are reported for these model coefficients. I did verify, however, that the hypothesis that 
Table 1: Data of the fish patty experiment.

\begin{tabular}{ccccccc|ccccccc}
\hline$z_{1}$ & $z_{2}$ & $z_{3}$ & $x_{1}$ & $x_{2}$ & $x_{3}$ & $y$ & $z_{1}$ & $z_{2}$ & $z_{3}$ & $x_{1}$ & $x_{2}$ & $x_{3}$ & $y$ \\
\hline 375 & 25 & 25 & 1 & 0 & 0 & 1.84 & 375 & 25 & 40 & 1 & 0 & 0 & 1.65 \\
375 & 25 & 25 & 0 & 1 & 0 & 0.67 & 375 & 25 & 40 & 0 & 1 & 0 & 0.58 \\
375 & 25 & 25 & 0 & 0 & 1 & 1.51 & 375 & 25 & 40 & 0 & 0 & 1 & 1.21 \\
375 & 25 & 25 & $1 / 2$ & $1 / 2$ & 0 & 1.29 & 375 & 25 & 40 & $1 / 2$ & $1 / 2$ & 0 & 1.18 \\
375 & 25 & 25 & $1 / 2$ & 0 & $1 / 2$ & 1.42 & 375 & 25 & 40 & $1 / 2$ & 0 & $1 / 2$ & 1.45 \\
375 & 25 & 25 & 0 & $1 / 2$ & $1 / 2$ & 1.61 & 375 & 25 & 40 & 0 & $1 / 2$ & $1 / 2$ & 1.07 \\
375 & 25 & 25 & $1 / 3$ & $1 / 3$ & $1 / 3$ & 1.59 & 375 & 25 & 40 & $1 / 3$ & $1 / 3$ & $1 / 3$ & 1.41 \\
425 & 25 & 25 & 1 & 0 & 0 & 2.86 & 425 & 25 & 40 & 1 & 0 & 0 & 2.32 \\
425 & 25 & 25 & 0 & 1 & 0 & 1.10 & 425 & 25 & 40 & 0 & 1 & 0 & 0.97 \\
425 & 25 & 25 & 0 & 0 & 1 & 1.60 & 425 & 25 & 40 & 0 & 0 & 1 & 2.12 \\
425 & 25 & 25 & $1 / 2$ & $1 / 2$ & 0 & 1.53 & 425 & 25 & 40 & $1 / 2$ & $1 / 2$ & 0 & 1.45 \\
425 & 25 & 25 & $1 / 2$ & 0 & $1 / 2$ & 1.81 & 425 & 25 & 40 & $1 / 2$ & 0 & $1 / 2$ & 1.93 \\
425 & 25 & 25 & 0 & $1 / 2$ & $1 / 2$ & 1.50 & 425 & 25 & 40 & 0 & $1 / 2$ & $1 / 2$ & 1.28 \\
425 & 25 & 25 & $1 / 3$ & $1 / 3$ & $1 / 3$ & 1.68 & 425 & 25 & 40 & $1 / 3$ & $1 / 3$ & $1 / 3$ & 1.54 \\
375 & 40 & 25 & 1 & 0 & 0 & 3.01 & 375 & 40 & 40 & 1 & 0 & 0 & 3.04 \\
375 & 40 & 25 & 0 & 1 & 0 & 1.21 & 375 & 40 & 40 & 0 & 1 & 0 & 1.16 \\
375 & 40 & 25 & 0 & 0 & 1 & 2.32 & 375 & 40 & 40 & 0 & 0 & 1 & 2.00 \\
375 & 40 & 25 & $1 / 2$ & $1 / 2$ & 0 & 1.93 & 375 & 40 & 40 & $1 / 2$ & $1 / 2$ & 0 & 1.85 \\
375 & 40 & 25 & $1 / 2$ & 0 & $1 / 2$ & 2.57 & 375 & 40 & 40 & $1 / 2$ & 0 & $1 / 2$ & 2.39 \\
375 & 40 & 25 & 0 & $1 / 2$ & $1 / 2$ & 1.83 & 375 & 40 & 40 & 0 & $1 / 2$ & $1 / 2$ & 1.60 \\
375 & 40 & 25 & $1 / 3$ & $1 / 3$ & $1 / 3$ & 1.94 & 375 & 40 & 40 & $1 / 3$ & $1 / 3$ & $1 / 3$ & 2.05 \\
425 & 40 & 25 & 1 & 0 & 0 & 4.13 & 425 & 40 & 40 & 1 & 0 & 0 & 4.13 \\
425 & 40 & 25 & 0 & 1 & 0 & 1.67 & 425 & 40 & 40 & 0 & 1 & 0 & 1.30 \\
425 & 40 & 25 & 0 & 0 & 1 & 2.57 & 425 & 40 & 40 & 0 & 0 & 1 & 2.75 \\
425 & 40 & 25 & $1 / 2$ & $1 / 2$ & 0 & 2.26 & 425 & 40 & 40 & $1 / 2$ & $1 / 2$ & 0 & 2.06 \\
425 & 40 & 25 & $1 / 2$ & 0 & $1 / 2$ & 3.15 & 425 & 40 & 40 & $1 / 2$ & 0 & $1 / 2$ & 2.82 \\
425 & 40 & 25 & 0 & $1 / 2$ & $1 / 2$ & 2.22 & 425 & 40 & 40 & 0 & $1 / 2$ & $1 / 2$ & 2.10 \\
425 & 40 & 25 & $1 / 3$ & $1 / 3$ & $1 / 3$ & 2.60 & 425 & 40 & 40 & $1 / 3$ & $1 / 3$ & $1 / 3$ & 2.32 \\
\hline & & & & & & & & & & & & &
\end{tabular}


$\beta_{1}^{\mathrm{m}}=\beta_{2}^{\mathrm{m}}=\beta_{3}^{\mathrm{m}}$ could be rejected in each of the reported models.

Note that a coefficient $\beta_{i}^{\mathrm{m}}$ represents the firmness of a patty consisting of the $i$ th fish species only, when the process variables act at their middle level and thus $z_{1}=z_{2}=z_{3}=0$. These coefficients should not be called main effects. Likewise, the coefficients $\beta_{i j}^{\mathrm{m}}$ are not referred to as interaction effects. Instead, the terms $\beta_{i j}^{\mathrm{m}} x_{i} x_{j}$ are named binary blending terms. Finally, the MPV model above does not involve main-effects terms for the process variables. This is because it would become inestimable due to perfect collinearity.

\section{Cumbersome complete randomization}

A complete randomization of the 56 tests in the fish patty experiment would require the experimenter to conduct the following steps 56 times:

1. make up an individual patty;

2. cook the patty individually at a selected temperature for a specific length of time;

3. freeze the patty individually; and

4. deep fat fry the patty individually for a particular length of time.

The use of complete randomization ensures that the observations are uncorrelated, so that the data can be analyzed using ordinary least squares.

Implying that complete randomization is cumbersome because it involves much repetitive work, Cornell (1988) then sketched two different logistical scenarios, each of which requires a split-plot data analysis. Split-plot experimental designs are often used due to logistical reasons. Goos and Jones (2011), for instance, explain that experiments spanning two steps of a production process, experiments involving prototypes, and experiments involving mixtures and process variables are often run using a split-plot design. A key feature of split-plot experiments is that the data involve groups of correlated observations. For this reason, the data from split-plot experiments have to be analyzed using generalized least squares rather than ordinary least squares. 


\section{Split-Plot Scenario 1}

\subsection{Logistics}

In the first split-plot scenario sketched by Cornell (1988), the experiment started by producing one patty of each of the seven blends defined by the simplex-centroid design. This resulted in seven different patties, which were cooked together at a selected temperature for a specific length of time, frozen and deep fat fried together. In this approach, the seven patties were processed simultaneously and therefore led to a group of seven correlated observations.

Next, seven new patties were individually prepared from scratch, again according to the simplex-centroid design. The seven new patties were then processed together, using a specific cooking temperature, a specific cooking time, and a specific deep fat frying time. The seven new patties then gave rise to a second group of seven correlated observations.

This procedure was repeated until all $2^{3}=8$ processing conditions had been tested, resulting in eight groups of seven correlated observations. A graphical display of the procedure is given in Figure 1. The eight processing conditions, represented by the vertices of the cube, are visited one by one, in a random order. At each vertex, seven individually prepared patties are subjected simultaneously to the corresponding processing condition.

In design of experiments jargon, the eight groups are the whole plots of a split-plot design, while the individual patties in each group are sub-plots. The groups are essentially larger experimental units, to which the processing conditions are applied. The individual patties are smaller experimental units, to which the different mixtures are applied. The process variables (cooking temperature, cooking time and deep fat frying time) are the whole-plot factors of the experiment, while the mixture ingredient proportions (sheepshead, mullet and croaker) are the sub-plot factors. In summary, this first scenario resulted in a split-plot design with eight whole plots containing seven subplots each.

In this scenario, every processing condition was run once, yielding a total of eight units of information concerning the process variables (cooking temperature, cooking time and deep fat frying time). In contrast, 56 mixture preparations were performed, each to create one patty. This yielded 56 units of information concerning the mixture ingredient proportions. 


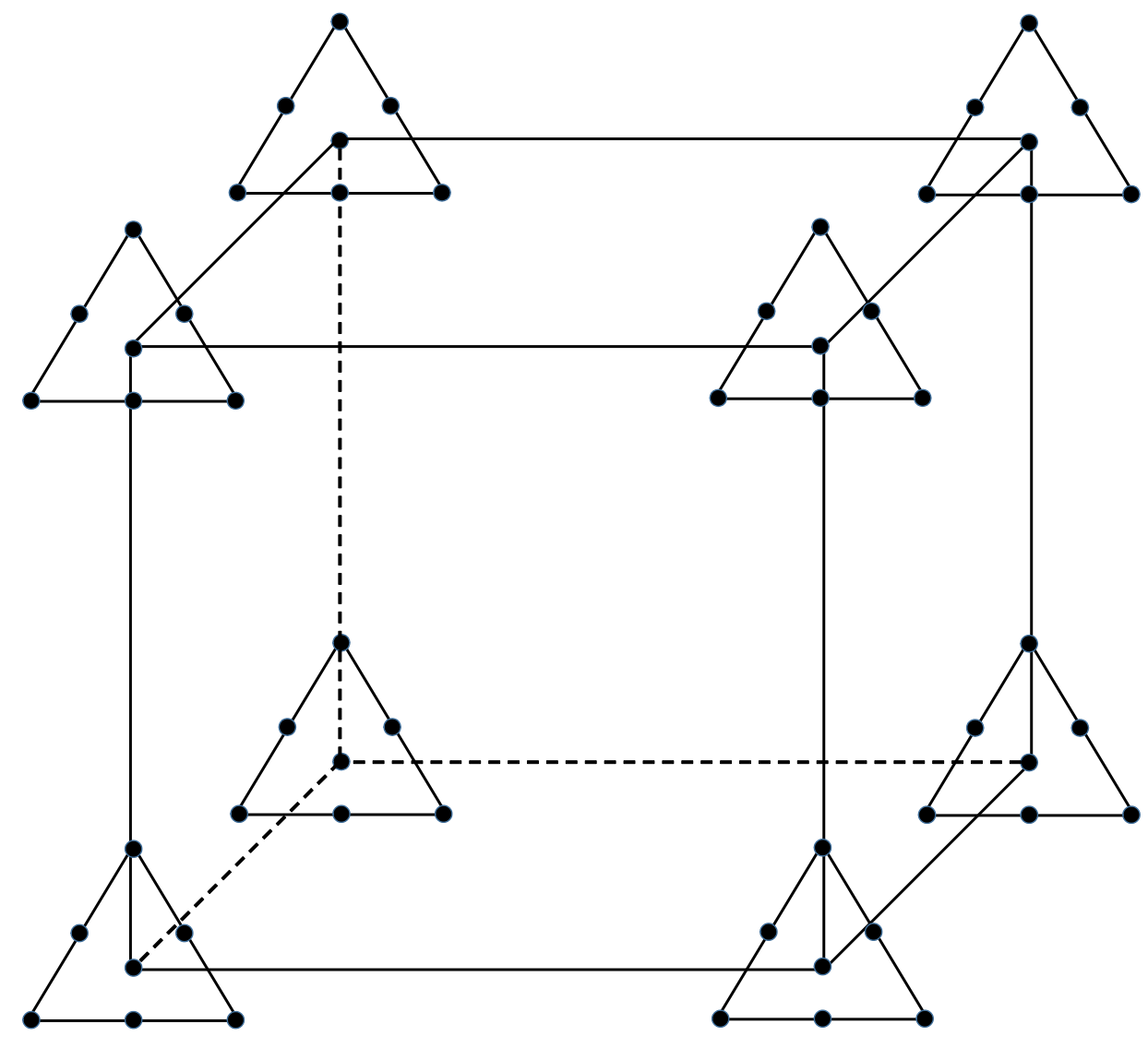

Figure 1: Schematic representation of Split-Plot Scenario 1 for the fish patty experiment. The vertices of the cube represent the $2^{3}$ combinations of the process variables (cooking temperature, cooking time and frying time). These combinations are visited one by one. At each combination, seven individually prepared patties, corresponding to the seven blends of a simplex-centroid design, are processed simultaneously. 


\subsection{Model}

A general statistical model for the first split-plot scenario is given by

$$
Y_{i j}=\mathbf{f}^{T}\left(\mathbf{x}_{i j}, \mathbf{z}_{i}\right) \boldsymbol{\beta}+\gamma_{i}+\varepsilon_{i j},
$$

where

- $Y_{i j}$ is the firmness of the $j$ th patty in the $i$ th whole plot, i.e., at the $i$ th processing condition (cooking temperature, cooking time and frying time);

- $\mathbf{x}_{i j}$ is the 3-dimensional vector containing the proportions of the three fish species in the $j$ th patty at the $i$ th processing condition (whole plot);

- $\mathbf{z}_{i}$ is the 3-dimensional vector containing the settings of the three process variables (cooking temperature, cooking time and frying time) at the $i$ th processing condition (whole plot);

- $\mathbf{f}^{T}\left(\mathbf{x}_{i j}, \mathbf{z}_{i}\right)$ denotes the model expansion of the three mixture ingredient proportions and the settings of the three process variables (see Equation (1) for the terms contained within $\left.\mathbf{f}^{T}\left(\mathbf{x}_{i j}, \mathbf{z}_{i}\right)\right)$;

- $\boldsymbol{\beta}$ is the vector containing the effects of the ingredient proportions and the process variables;

- $\gamma_{i}$ is the whole-plot error, i.e., the random error in the firmness response associated with the $i$ th processing condition (whole plot); and

- $\varepsilon_{i j}$ is the sub-plot error, i.e., the random error in the firmness response associated with the $j$ th patty at the $i$ th processing condition (whole plot).

Note that, in the model for Split-Plot Scenario 1, the vector containing the settings for the process variables, $\mathbf{z}_{i}$, only has index $i$ because the settings remain fixed while processing the seven patties in whole plot $i$. In contrast, the vector with mixture ingredient proportions, $\mathbf{x}_{i j}$, has two indices because the mixture is varied from observation to observation and every observation is uniquely identified only by the whole-plot and the sub-plot indices. The whole-plot errors $\gamma_{i}$ and the sub-plot errors $\varepsilon_{i j}$ are all random variables assumed to be independently normally distributed with zero mean. 
The whole-plot errors $\gamma_{i}$ are assumed to have a common variance, $\sigma_{\gamma}^{2}$, while the sub-plot errors $\varepsilon_{i j}$ are assumed to have common variance $\sigma_{\varepsilon}^{2}$. The sum $\sigma_{\gamma}^{2}+\sigma_{\varepsilon}^{2}$ represents the total random variation in the firmness of the patties, while $\rho=\sigma_{\gamma}^{2} /\left(\sigma_{\gamma}^{2}+\sigma_{\varepsilon}^{2}\right)$ is the correlation in the firmness within each group of seven patties processed simultaneously (i.e., within each whole plot). This is because, in this first split-plot model, the random term $\gamma_{i}$ captures the similarity between the responses of all seven tests carried out simultaneously at the $i$ th processing condition.

Due to the correlated nature of the observations performed at a given processing condition in Split-Plot Scenario 1, generalized rather than ordinary least squares should be used for estimating the effects of the ingredient proportions and the process variables in $\boldsymbol{\beta}$. The variance components $\sigma_{\varepsilon}^{2}$ and $\sigma_{\gamma}^{2}$ can be estimated using restricted maximum likelihood, and standard errors and degrees of freedom for significance testing can be obtained using the method of Kenward and Roger (1997). Details about the analysis of split-plot data can be found in Letsinger et al. (1996), Goos et al. (2006) and Goos and Jones (2011). Note that, for specific experimental designs such as crossed split-plot designs, the point estimates produced by ordinary and generalized least squares coincide. The design for the fish patty experiment under Split-Plot Scenario 1 belongs to the class of crossed split-plot designs, as a result of which the same point estimates are obtained with ordinary and generalized least squares. However, the standard errors and degrees of freedom for hypothesis testing strongly depend on the method of analysis, and the significances may therefore differ too.

\subsection{Analysis results}

Analyzing the data from the fish patty experiment under Split-Plot Scenario 1 indicated that

- the coefficients of the three ingredient proportions in the model were significantly different from each other, implying that the firmnesses of the pure ingredients differed significantly at intermediate levels of the cooking temperature, cooking time and frying time (whose coded values are zero);

- two of the three coefficients of the binary blending terms were significantly different from zero; 
Table 2: Details for the final model selected under Split-Plot Scenario 1, in which the process variables (cooking temperature, cooking time and frying time) act as whole-plot factors in a split-plot MPV experiment.

\begin{tabular}{lrccrl}
\hline Source & Estimate & St. Err. & DF & $t$ Ratio & $p$ Value \\
\hline Fish 1 & 2.862 & 0.070 & 37.28 & & \\
Fish 2 & 1.124 & 0.065 & 33.40 & & \\
Fish 3 & 2.052 & 0.065 & 33.40 & & \\
Fish 1*Fish 2 & -1.034 & 0.300 & 40.00 & -3.45 & 0.0013 \\
Fish 1*Fish 3 & -0.894 & 0.300 & 40.00 & -2.98 & 0.0049 \\
Fish 1*Cooking Temperature & 0.383 & 0.059 & 28.39 & 6.45 & $<.0001$ \\
Fish 2*Cooking Temperature & 0.091 & 0.059 & 28.39 & 1.52 & 0.1386 \\
Fish 3*Cooking Temperature & 0.190 & 0.059 & 28.39 & 3.20 & 0.0034 \\
Fish 1*Cooking Time & 0.649 & 0.059 & 28.39 & 10.92 & $<.0001$ \\
Fish 2*Cooking Time & 0.186 & 0.059 & 28.39 & 3.12 & 0.0041 \\
Fish 3*Cooking Time & 0.387 & 0.059 & 28.39 & 6.51 & $<.0001$ \\
\hline
\end{tabular}

- frying time had no significant effect on the firmness;

- cooking time and cooking temperature each had a significant positive effect on the firmness, but the strength of the effect varied significantly with the proportions of the three ingredients; and

- there were no significant interaction effects among the process variables.

The parameter estimates in the final model selected are shown in Table 2 , along with their standard errors, the denominator degrees of freedom, the $t$ ratios and the $p$ values. Cooking time and temperature have positive effects on the patties' firmness, but the strengths of the effects depend on the fish mixtures utilized. The estimated effect of cooking time varies from 0.186 to 0.649 depending on the fish mixture used, while the estimated effect of cooking temperature varies from 0.091 to 0.383 . Combining the first fish (mullet) with the second (sheepshead), and the first (mullet) with the third (croaker) caused the patties to be softer.

The variances $\sigma_{\gamma}^{2}$ and $\sigma_{\varepsilon}^{2}$ were estimated to be 0.005 and 0.034 , respectively, when the final model was fitted. The correlation $\rho$ was estimated to be 0.128 . The firmnesses of the seven patties processed together are thus slightly correlated. 


\section{Split-Plot Scenario 2}

\subsection{Logistics}

In the second split-plot scenario sketched by Cornell (1988), the experiment started by randomly selecting one of the seven mixtures from the simplexcentroid design and preparing a batch of that mixture. From that batch, eight patties were produced. Each of the eight patties was then subjected to one of the processing conditions defined by the $2^{3}$ factorial design. Since the patties originated from the same batch, they gave rise to a group of eight correlated observations, despite the fact that they were processed individually.

Next, a different mixture from the simplex-centroid design was selected at random and a batch of that mixture was prepared. From that second batch, eight patties were produced, and each of the eight patties was subjected to a second one of the processing conditions defined by the $2^{3}$ factorial design. Since the eight new patties all originated from the same batch, they also gave rise to a group of eight correlated observations.

This procedure was repeated until a batch had been prepared for each of the seven mixtures from the simplex-centroid design, and the $2^{3}=8$ processing conditions had been tested individually for the eight patties produced from each batch. The procedure therefore resulted in seven groups of eight correlated observations. A graphical display of the procedure is given in Figure 2. The seven mixtures, represented by the vertices, edge centroids and overall centroid of the triangle in the figure, are visited one by one, and a batch is made for each of them. Eight patties are made from each batch to test the eight processing conditions individually.

This time, the seven batches act as the whole plots of a split-plot design, while the processing conditions correspond to the sub-plots. The batches are essentially larger experimental units, to which the mixtures are assigned. The individual patties created from the batches are smaller experimental units, to which the different processing conditions are applied. This time, the whole-plot factors are the three mixture ingredient proportions, while the process variables (cooking temperature, cooking time and deep fat frying time) are the sub-plot factors of the experiment.

In this second split-plot scenario, each of the $2^{3}$ processing conditions was run independently seven times, yielding a total of 56 units of information concerning the process variables (cooking temperature, cooking time and deep fat frying time). In contrast, mixtures were produced on only seven 


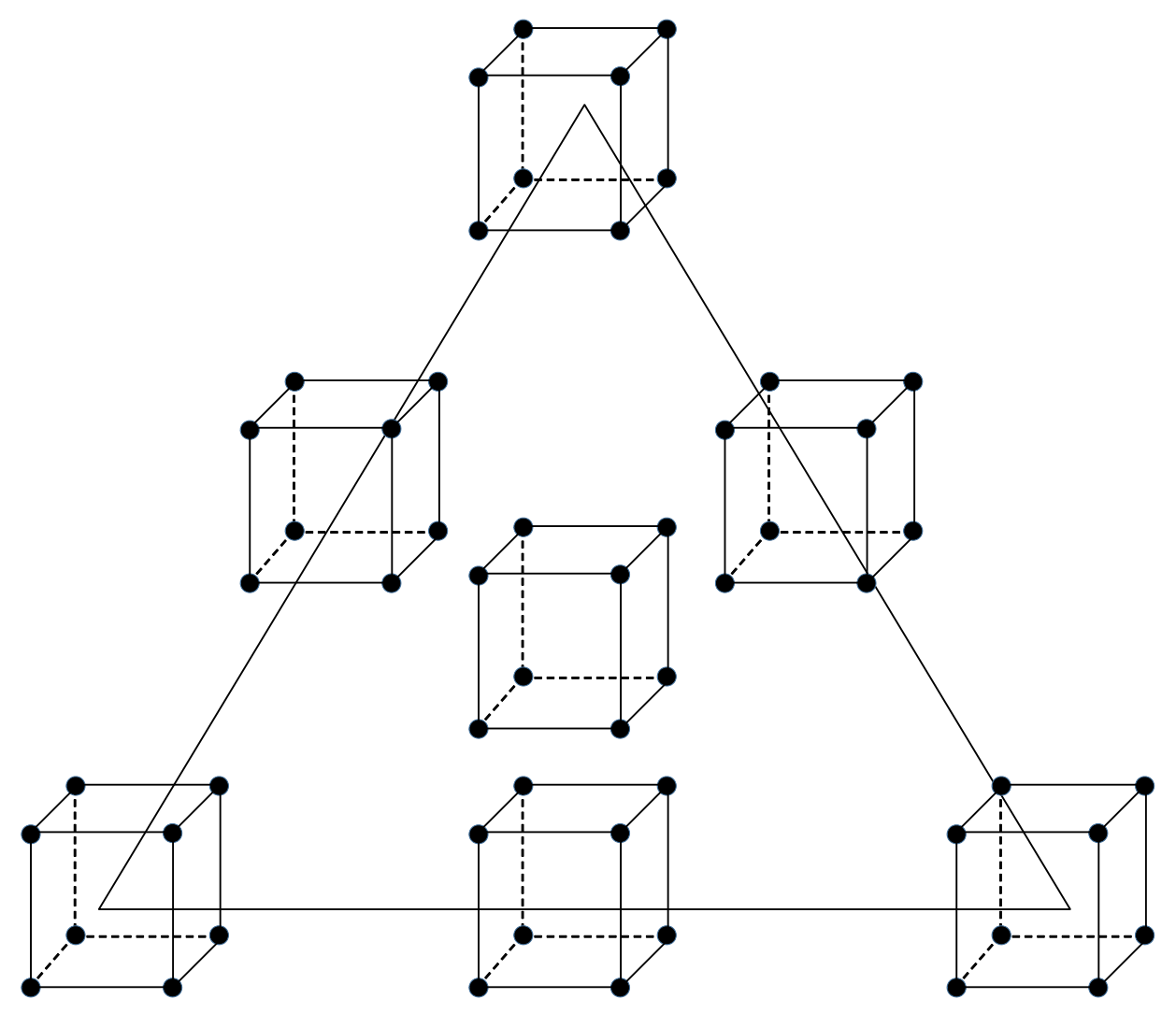

Figure 2: Schematic representation of Split-Plot Scenario 2 for the fish patty experiment. The positions of the seven cubes correspond to the fish blends of a simplex-centroid design. For each of the seven blends, a batch of eight patties is prepared. The eight patties are then processed individually at the $2^{3}$ different processing conditions, represented by the vertices of the cube corresponding to the blend. 
occasions. This yielded merely seven units of information concerning the mixture ingredient proportions.

\subsection{Model}

A general statistical model for the second split-plot scenario is given by

$$
Y_{i j}=\mathbf{f}^{T}\left(\mathbf{x}_{i}, \mathbf{z}_{i j}\right) \boldsymbol{\beta}+\gamma_{i}+\varepsilon_{i j},
$$

where

- $Y_{i j}$ is the firmness of the $j$ th patty from the $i$ th whole plot, i.e., the patty produced from the $i$ th batch and processed individually using the $j$ th processing condition;

- $\mathbf{x}_{i}$ is the 3-dimensional vector containing the proportions of the three fish species in the $i$ th batch (whole plot);

- $\mathbf{z}_{i j}$ is the 3-dimensional vector containing the settings of the three process variables (cooking temperature, cooking time and frying time) used for the $j$ th patty from the $i$ th batch (whole plot);

- $\mathbf{f}^{T}\left(\mathbf{x}_{i}, \mathbf{z}_{i j}\right)$ denotes the model expansion of the three mixture ingredient proportions and the settings of the three process variables (see Equation (1) for the terms contained within $\left.\mathbf{f}^{T}\left(\mathbf{x}_{i}, \mathbf{z}_{i j}\right)\right)$;

- $\boldsymbol{\beta}$ is the vector containing the effects of the ingredient proportions and process variables;

- $\gamma_{i}$ is the whole-plot error, i.e., the random error in the firmness response associated with the $i$ th batch (whole plot); and

- $\varepsilon_{i j}$ is the sub-plot error, i.e., the random error in the firmness response associated with the $j$ th patty created from the $i$ th batch and processed individually at the $j$ th processing condition.

In the model for Split-Plot Scenario 2, the vector containing the mixture ingredient proportions, $\mathbf{x}_{i}$, only has index $i$ because the ingredient proportions are fixed for all the observations in whole plot $i$. In contrast, the vector with the settings of the process variables, $\mathbf{z}_{i j}$, has two indices because the processing condition is varied from observation to observation and every observation 
is uniquely identified only by the whole-plot and the sub-plot indices. The random errors $\gamma_{i}$ and $\varepsilon_{i j}$ are again assumed to be independently normally distributed with zero mean and variances $\sigma_{\gamma}^{2}$ and $\sigma_{\varepsilon}^{2}$, respectively.

In this second split-plot model, the random term $\gamma_{i}$ captures the similarity in the responses of all observations conducted using patties originating from the $i$ th batch (whole plot). Therefore, this time, $\rho=\sigma_{\gamma}^{2} /\left(\sigma_{\gamma}^{2}+\sigma_{\varepsilon}^{2}\right)$ is the correlation in the firmness within each group of eight patties originating from a given batch.

Due to the correlated nature of the observations obtained from the same preparation of a given mixture in Split-Plot Scenario 2, generalized rather than ordinary least squares should be used for estimating the effects in $\boldsymbol{\beta}$ here as well, the variance components $\sigma_{\varepsilon}^{2}$ and $\sigma_{\gamma}^{2}$ can be estimated using restricted maximum likelihood, and standard errors and degrees of freedom for significance testing can be obtained from Kenward and Roger (1997). Since the design for the fish patty experiment under Split-Plot Scenario 2 also belongs to the class of crossed split-plot designs, the same point estimates for the effects in $\boldsymbol{\beta}$ are obtained with ordinary and generalized least squares. However, the standard errors, the degrees of freedom for hypothesis testing and the significances again depend on the method of analysis.

\subsection{Analysis results}

Analyzing the data from the fish patty experiment under Split-Plot Scenario 2 indicated that

- the coefficients of the three ingredient proportions in the model were significantly different from each other, implying that the firmnesses of the pure ingredients differed significantly at intermediate levels of the cooking temperature, cooking time and frying time (whose coded values are zero);

- none of the three coefficients of the binary blending terms were significantly different from zero;

- all three process variables (cooking temperature, cooking time and frying time) had significant effects on the firmness of the patties;

- the effects of cooking time and cooking temperature varied significantly with the proportions of the three ingredients; 
Table 3: Details for the final model selected under Split-Plot Scenario 2, in which the mixture ingredient proportions act as whole-plot factors in a split-plot MPV experiment.

\begin{tabular}{lcccrc}
\hline Term & Estimate & Std Error & DF & $t$ Ratio & $p$ Value \\
\hline Fish 1 & 2.699 & 0.133 & 4 & & \\
Fish 2 & 1.050 & 0.133 & 4 & & \\
Fish 3 & 1.992 & 0.133 & 4 & & \\
Fish 1*Cooking Temperature & 0.383 & 0.052 & 42 & 7.43 & 0.0000 \\
Fish 2*Cooking Temperature & 0.091 & 0.052 & 42 & 1.76 & 0.0864 \\
Fish 3*Cooking Temperature & 0.190 & 0.052 & 42 & 3.68 & 0.0007 \\
Fish 1*Cooking Time & 0.649 & 0.052 & 42 & 12.58 & 0.0000 \\
Fish 2*Cooking Time & 0.186 & 0.052 & 42 & 3.60 & 0.0008 \\
Fish 3*Cooking Time & 0.387 & 0.052 & 42 & 7.50 & 0.0000 \\
Frying time & -0.066 & 0.024 & 42 & -2.78 & 0.0081 \\
\hline
\end{tabular}

- the effect of frying time did not differ significantly across the various fish mixtures; and

- there were no significant interaction effects among the process variables.

The parameter estimates in the final model selected for Split-Plot Scenario 2 are shown in Table 3, along with their standard errors, the denominator degrees of freedom, the $t$ ratios and the $p$ values. The cooking time and temperature have positive effects on the patties' firmnesses, but the strengths of the effects depend on the fish mixture utilized. Again, the estimated effect of cooking time varies from 0.186 to 0.649 depending on the fish mixture used, while the estimated effect of cooking temperature varies from 0.091 to 0.383 . The frying time has a small but statistically significant negative effect on the firmness, but there is no evidence that this effect differs from one fish mixture to another.

The variances $\sigma_{\gamma}^{2}$ and $\sigma_{\varepsilon}^{2}$ were estimated to be 0.022 and 0.031 , respectively, when the final model was fitted for Split-Plot Scenario 2. The correlation $\rho$ was therefore estimated to be 0.415 . The firmnesses of the eight patties originating from a given batch in Split-Plot Scenario 2 were therefore more strongly correlated than the firmnesses of the patties processed together under Split-Plot Scenario 1. 


\section{Discussion of the two split-plot analyses}

The two split-plot analyses exhibit similarities as well as differences. Both analyses indicate that the ingredient proportions matter, that cooking time and cooking temperature have positive effects on the patties' firmnesses, and that the effects of these two process variables differ from one mixture to another. That the ingredient proportions matter can be seen in various ways. First, the null hypothesis that the coefficients of the three ingredient proportions in the model are equal was rejected. Second, modifying the ingredient proportions influences the effects of cooking temperature and time. Third, in Split-Plot Scenario 1, two of the binary blending terms appear in the final model.

The second split-plot analysis suggests that the main effect of the process variable frying time is significant, while the first does not. As shown in Table 3, the point estimate for that main effect is -0.066 in the final model for Split-Plot Scenario 2. Adding the main effect of frying time to the final model for Split-Plot Scenario 1 produces the same point estimate, but the degrees of freedom associated with the effect are so low in Split-Plot Scenario 1 that it is not significant. The degrees of freedom for the main effect of frying time are 4 in Split-Plot Scenario 1 (where each processing condition is tested only once per mixture formulation) and 42 in Split-Plot Scenario 2 (where each processing condition is used seven times). The large difference in degrees of freedom impacts the widths of the confidence intervals for the main effect in the two analyses. When adding the main effect of frying time to the final model in the first split-plot analysis, the standard error is 0.0216, and its $99 \%$ confidence interval is $[-0.165,0.034]$. In the second split-plot analysis, the standard error for the main effect of frying time is 0.0237 , and the $99 \%$ confidence interval is $[-0.130,-0.002]$. Thus, due to the larger degrees of freedom in Split-Plot Scenario 2, the confidence interval for the main effect of frying time is narrower, despite the larger standard error in that scenario. This shows how large the influence of the specific split-plot experimental design and the corresponding analysis on the statistical inference can be.

When looking at the $p$ values, the binary blending terms are not significant in the second split-plot analysis, while two of these three terms are significant in the first split-plot analysis. In the second split-plot analysis, the point estimate for the coefficient of the binary blending term involving the first fish (mullet) and the second (sheepshead) would be -1.034 , with a $99 \%$ confidence interval equal to $[-5.522,3.455]$, while the coefficient 
of the binary blending term involving the first fish (mullet) and the third (croaker) would be estimated to be -0.894 , with $99 \%$ confidence interval $[-5.382,3.595]$, when adding the two binary blending terms to the model. The second split-plot analysis therefore suggests that the two coefficients of the binary blending terms most likely are negative, but there is substantial uncertainty. The uncertainty is so large that the coefficients may in fact be quite large and positive, instead of negative. The first split-plot analysis produces the same point estimates for both coefficients, but much narrower 99\% confidence intervals: $[-1.844,-0.223]$ and $[-1.704,-0.083]$. The massive differences in confidence interval widths are due to the large differences in degrees of freedom (2 versus 40) and standard errors (0.453 versus 0.300 ).

Comparing the point estimates for Split-Plot Scenario 1 and Split-Plot Scenario 2 in Tables 2 and 3 shows that, for certain model terms, the estimates match. This is due to the fact that the designs for the fish patty experiment are crossed split-plot designs in both scenarios. That not all the estimates match is caused by the fact that the final models selected for the two scenarios do not involve the same terms.

\section{Strip-Plot Scenario}

\subsection{Logistics}

Looking at the fish patty data under the two different split-plot scenarios discussed by Cornell (1988) leads to different sets of statistically significant effects and different models. Obviously, it makes sense from a logistical point of view to process multiple patties at the same time for each given processing condition, as in Split-Plot Scenario 1. It also makes sense to prepare enough of each fish mixture to produce multiple patties from each given preparation, as in Split-Plot Scenario 2. However, the most convenient way to conduct the fish patty experiment is to process multiple patties simultaneously (as in Split-Plot Scenario 1) and to prepare enough of each mixture to produce multiple patties from each preparation (as in Split-Plot Scenario 2). In this combined approach, the observations are arranged in groups in two ways, according to the batches and according to the processing conditions. The combined approach is depicted schematically in Table 4 and involves the following steps:

1. In Step 1, seven batches of fish mixtures can be prepared, one for each 
Table 4: Schematic representation of the strip-plot approach to conducting the fish patty experiment.

\begin{tabular}{|c|c|c|c|c|c|c|c|c|c|c|}
\hline & \multirow{3}{*}{ Batch } & \multirow{3}{*}{$\begin{array}{l}\text { Ingredient } \\
\text { Proportions }\end{array}$} & \multirow{2}{*}{\multicolumn{8}{|c|}{$\begin{array}{c}\text { Steps } 3 \& 4 \\
\text { Set }+ \text { Processing Condition }\end{array}$}} \\
\hline & & & & & & & & & & \\
\hline & & & 1 & 2 & 3 & 4 & 5 & 6 & 7 & 8 \\
\hline $\mathrm{S}$ & 1 & $(1,0,0)$ & $\mathrm{P}_{11}$ & $\mathrm{P}_{12}$ & $\mathrm{P}_{13}$ & $\mathrm{P}_{14}$ & $\mathrm{P}_{15}$ & $\mathrm{P}_{16}$ & $\mathrm{P}_{17}$ & $\mathrm{P}_{18}$ \\
\hline $\mathrm{t}$ & 2 & $(0,1$ & $\mathrm{P}_{21}$ & $\mathrm{P}_{22}$ & $\mathrm{P}_{23}$ & $\mathrm{P}_{24}$ & $\mathrm{P}_{25}$ & $\mathrm{P}_{26}$ & $\mathrm{P}_{27}$ & $\mathrm{P}_{28}$ \\
\hline $\mathrm{e}$ & 3 & $(0,0,1$ & $P_{31}$ & $\mathrm{P}_{32}$ & $\mathrm{P}_{33}$ & $\mathrm{P}_{34}$ & $\mathrm{P}_{35}$ & $\mathrm{P}_{36}$ & $\mathrm{P}_{37}$ & $\mathrm{P}_{38}$ \\
\hline $\mathrm{p}$ & 4 & $(1 /$ & $\mathrm{P}_{41}$ & $\mathrm{P}_{42}$ & $\mathrm{P}_{43}$ & $\mathrm{P}_{44}$ & $\mathrm{P}_{45}$ & $\mathrm{P}_{46}$ & $\mathrm{P}_{47}$ & $\mathrm{P}_{48}$ \\
\hline $\mathrm{s}$ & 5 & $(1 / 2$, & $\mathrm{P}_{51}$ & $\mathrm{P}_{52}$ & $\mathrm{P}_{53}$ & $\mathrm{P}_{54}$ & $\mathrm{P}_{55}$ & $\mathrm{P}_{56}$ & $\mathrm{P}_{57}$ & $\mathrm{P}_{58}$ \\
\hline & 6 & $(0,1 / 2,1 / 2)$ & $\mathrm{P}_{61}$ & $\mathrm{P}_{62}$ & $\mathrm{P}_{63}$ & $\mathrm{P}_{64}$ & $\mathrm{P}_{65}$ & $\mathrm{P}_{66}$ & $\mathrm{P}_{67}$ & $\mathrm{P}_{68}$ \\
\hline $1 \& 2$ & 7 & $(1 / 3,1 / 3,1 / 3)$ & $\mathrm{P}_{71}$ & $\mathrm{P}_{72}$ & $\mathrm{P}_{73}$ & $\mathrm{P}_{74}$ & $\mathrm{P}_{75}$ & $\mathrm{P}_{76}$ & $\mathrm{P}_{77}$ & $\mathrm{P}_{78}$ \\
\hline & & Cooking Temp. & 375 & 375 & 375 & 375 & 425 & 425 & 425 & 425 \\
\hline & & Cooking Tin & 25 & 25 & 40 & 40 & 25 & 25 & 40 & 40 \\
\hline & & Frying Time & 25 & 40 & 25 & 40 & 25 & 40 & 25 & 40 \\
\hline
\end{tabular}

mixture in the simplex-centroid design. The seven batches correspond to the rows in Table 4 .

2. In Step 2, from each of these seven batches, eight patties can be produced. This results in seven groups of eight patties. In Table 4, the eight patties produced from a given batch $i$ are labeled $\mathrm{P}_{i 1}, \mathrm{P}_{i 2}, \ldots$, $\mathrm{P}_{i 8}$.

3. In Step 3, eight sets of seven patties can be formed. Each set should contain one patty from each of the seven batches. In Table 4 , the $j$ th set contains the patties $\mathrm{P}_{1 j}, \mathrm{P}_{2 j}, \ldots, \mathrm{P}_{7 j}$. The eight sets of seven patties appear in the eight rightmost columns of the table.

4. In Step 4, for each of the eight sets of seven patties, one processing condition has to be chosen and all seven patties from that set can then be processed at that condition simultaneously. In Table 4, the processing condition assigned to each set is shown at the bottom of its column.

Since each group of eight patties, $\mathrm{P}_{i 1}, \mathrm{P}_{i 2}, \ldots, \mathrm{P}_{i 8}$, is produced from the same batch $i$, it will give rise to eight correlated observations. So, the batch-wise preparation causes a first kind of correlation in the observations. There will, 
however, also be a second kind of correlation: the observations coming from patties that are processed together at any processing condition $j$, namely $\mathrm{P}_{1 j}, \mathrm{P}_{2 j}, \ldots, \mathrm{P}_{7 j}$, will be correlated as well.

The new scenario for the fish patty experiment involves preparing seven mixtures only one time and running through the entire cooking and frying process only eight times. Clearly, compared to both split-plot scenarios and to complete randomization, this is the more convenient approach. The experimental design corresponding to this third scenario is generally called a strip-plot design, but the names two-way split-plot design, strip-block design or criss-cross design are also utilized (Goos and Jones, 2011). Box and Jones (1992) wrote an instructive article about the use of split-plot and strip-plot designs for industrial experiments. While the strip-plot design is logistically convenient, it does have a few downsides too. I discuss these downsides in Section 7.

\subsection{Model}

A general statistical model for the Strip-Plot Scenario is given by

$$
Y_{i j}=\mathbf{f}^{T}\left(\mathbf{x}_{i}, \mathbf{z}_{j}\right) \boldsymbol{\beta}+\gamma_{i}+\delta_{j}+\varepsilon_{i j},
$$

where

- $Y_{i j}$ is the firmness of the patty produced from the $i$ th batch and processed at the $j$ th processing condition;

- $\mathbf{x}_{i}$ is the 3-dimensional vector containing the proportions of the three fish species in the $i$ th batch;

- $\mathbf{z}_{j}$ is the 3-dimensional vector containing the settings of the three process variables (cooking temperature, cooking time and frying time) at the $j$ th processing condition;

- $\mathbf{f}^{T}\left(\mathbf{x}_{i}, \mathbf{z}_{j}\right)$ denotes the model expansion of the three mixture ingredient proportions and the settings of the three process variables (see Equation (1) for the terms contained within $\left.\mathbf{f}^{T}\left(\mathbf{x}_{i}, \mathbf{z}_{j}\right)\right)$;

- $\boldsymbol{\beta}$ is the vector containing the effects of the ingredient proportions and process variables; 
- $\gamma_{i}$ is the random error in the firmness response due to the preparation of the $i$ th batch;

- $\delta_{j}$ is the random error in the firmness response associated with the application of the $j$ th processing condition; and

- $\varepsilon_{i j}$ is the random error in the firmness response for the patty created from the $i$ th batch and processed at the $j$ th processing condition.

In this strip-plot model, the random terms $\gamma_{i}$ and $\delta_{j}$ capture the correlations in the response values due to the two kinds of grouping in the data. It is assumed that all $\gamma_{i}, \delta_{j}$ and $\varepsilon_{i j}$ are independently normally distributed random variables with zero means and variances $\sigma_{\gamma}^{2}, \sigma_{\delta}^{2}$ and $\sigma_{\varepsilon}^{2}$, respectively. The correlation due to the batches is given by $\rho_{1}=\sigma_{\gamma}^{2} /\left(\sigma_{\gamma}^{2}+\sigma_{\delta}^{2}+\sigma_{\varepsilon}^{2}\right)$, and the correlation due to the simultaneous processing of each set of seven patties is $\rho_{2}=\sigma_{\delta}^{2} /\left(\sigma_{\gamma}^{2}+\sigma_{\delta}^{2}+\sigma_{\varepsilon}^{2}\right)$.

Obviously, the correlated nature of the observations in the Strip-Plot Scenario also necessitates the use of generalized least squares for estimating the effects in $\boldsymbol{\beta}$. Restricted maximum likelihood can be used for estimating the variance components $\sigma_{\gamma}^{2}, \sigma_{\delta}^{2}$ and $\sigma_{\varepsilon}^{2}$, and the method of Kenward and Roger (1997) can be used for determining the standard errors and degrees of freedom for significance testing.

\subsection{Analysis results}

Analyzing the data from the fish patty experiment under the strip-plot scenario indicated that

- the coefficients of the three ingredient proportions in the model were significantly different from each other, implying that the firmnesses of the pure ingredients differed significantly at intermediate levels of the cooking temperature, cooking time and frying time (whose coded values are zero);

- none of the three coefficiencts of the binary blending terms were significantly different from zero;

- two of the three process variables (cooking time and cooking temperature) had significant effects on the firmness of the patties; 
Table 5: Details for the final model selected under the strip-plot scenario discussed in Section 6

\begin{tabular}{lcccrc}
\hline Term & Estimate & Std Error & DF & $t$ Ratio & $p$ Value \\
\hline Fish 1 & 2.699 & 0.136 & 4.29 & & \\
Fish 2 & 1.050 & 0.136 & 4.29 & & \\
Fish 3 & 1.992 & 0.136 & 4.29 & & \\
Fish $1^{*}$ Cooking Temperature & 0.383 & 0.058 & 26.64 & 6.59 & 0.0000 \\
Fish $2^{*}$ Cooking Temperature & 0.091 & 0.058 & 26.64 & 1.56 & 0.1310 \\
Fish $3^{*}$ Cooking Temperature & 0.190 & 0.058 & 26.64 & 3.27 & 0.0030 \\
Fish $1^{*}$ Cooking Time & 0.649 & 0.058 & 26.64 & 11.17 & 0.0000 \\
Fish $2^{*}$ Cooking Time & 0.186 & 0.058 & 26.64 & 3.19 & 0.0036 \\
Fish $3^{*}$ Cooking Time & 0.387 & 0.058 & 26.64 & 6.66 & 0.0000 \\
\hline
\end{tabular}

- the effects of cooking time and cooking temperature varied significantly with the proportions of the three ingredients;

- the main effect of frying time was not significantly different from zero; and

- there were no significant interaction effects among the process variables.

The parameter estimates in the final model selected for the Strip-Plot Scenario are shown in Table 5, along with their standard errors, the denominator degrees of freedom, the $t$ ratios and the $p$ values. Again, the cooking time and temperature have positive effects on the patties' firmnesses, and the strengths of these effects depend on the fish mixture utilized.

The variances $\sigma_{\gamma}^{2}, \sigma_{\delta}^{2}$ and $\sigma_{\varepsilon}^{2}$ were estimated to be $0.022,0.005$ and 0.032 , respectively, when the final model was fitted for the strip-plot scenario. The correlation due to the batches, $\rho_{1}$, was therefore estimated to be 0.373 , while the correlation due to the patties being simultaneously processed under given conditions, $\rho_{2}$, was estimated to be 0.090 . The firmnesses of the eight patties originating from a given batch were therefore more strongly correlated than the firmnesses in the sets of patties processed together.

Of the three analyses presented here, the strip-plot analysis leads to the model with the fewest significant effects. In the strip-plot analysis, the binary blending terms are not statistically significant, and neither is the main effect of the frying time. This is because the strip-plot analysis accounts for only 
seven independent mixtures being prepared in the strip-plot scenario and for each of the eight processing conditions being run only once. This limits the amount of information concerning the effects of the mixture ingredients as well as the amount of information concerning the main effects of the process variables and the interactions involving process variables only. Hence, using a strip-plot design may result in failing to detect significant effects because of smaller degrees of freedom for more model terms. The only kinds of effects for which there are many degrees of freedom in the strip-plot analysis are the interactions involving a mixture ingredient proportion and a process variable.

\section{Discussion}

In this article, I have revisited the well-known data set from the fish patty experiment introduced in the literature by Cornell (1981) and Cornell and Gorman (1984). Cornell (1988) used the fish patty experiment as the motivating example for an article on the split-plot nature of many MPV experiments. In that article, he describes two alternative ways in which the experiment might have been performed. Both ways require a split-plot analysis of the data.

Cornell (1988), however, did not discuss the corresponding analyses of the fish patty data. In this article, I provided the split-plot analyses of the data for the two scenarios described by Cornell (1988). I also discussed a third alternative way in which the fish patty experiment might have been performed. This new scenario requires a strip-plot rather than a split-plot analysis. Therefore, in this article, I also discussed the strip-plot analysis of the fish patty data and contrasted it with the two split-plot analyses.

It is likely that certain MPV experiments are actually run using a stripplot design. Since that design is rather unknown, this may happen inadvertently. One example of a strip-plot MPV experiment can be found in Akkermans et al. (2017), who discussed excipient compatibility studies in the pharmaceutical industry and who pointed out that, depending on the logistics, these studies are either run as split-plot or strip-plot experiments. In any case, researchers analyzing data from MPV experiments should make sure that they carefully study the logistics of the experiment and perform the corresponding statistical analysis. If there is no grouping of the observations, the experiment was completely randomized and an ordinary least squares analysis is justified. If there is a single way in which the observations 
are grouped, a split-plot analysis is appropriate. If there are two ways in which the observations are grouped and the groups are crossed, a strip-plot analysis is required.

This article was written from the perspective of choosing the correct data analysis given that the experiment has already been run or the experimental design has already been selected. While the choice of experimental design is often driven by the logistics, it may, in some cases, be logistically feasible to conduct a MPV using (i) a completely randomized design, (ii) a splitplot design in which the process variables act as whole-plot factors (and the mixture ingredient proportions as sub-plot factors), (iii) a split-plot design in which the mixture ingredient proportions act as whole-plot factors (and the process variables as sub-plot factors) or (iv) a strip-plot design. Comparing these four choices to provide general recommendations is not a trivial task. First of all, it does not make sense to compare these design options assuming that they all involve the same number of observations. This is because performing a completely randomized MPV experiment is much more cumbersome that performing a split-plot or strip-plot MPV experiment.

For a sensible comparison of the design options, it is necessary to compare relatively large split-plot and strip-plot MPV designs with relatively small completely randomized designs. How much larger the split-plot and strip-plot designs should be depends on the practicalities, the logistics of the experiment and the cost of measuring the response for an individual observation. Second, the relative performance of the four design options depends on the magnitudes of the variance components, which themselves strongly depend on the application area.

Finally, the quality of a split-plot design strongly depends on the number of whole plots, and the quality of a strip-plot design strongly depends on the numbers of groups and sets. Therefore, the only general recommendation I can provide is that, for a given budget, a given availability of time and given resources, an experimenter should construct the largest possible completely randomized, split-plot and strip-plot designs feasible and evaluate these designs for given anticipated values of the variance components. Perhaps the most sensible way to evaluate the advantages and disadvantages of each design option then is to compare the powers for the various kinds of model effects.

The strenghts and weaknesses of split-plot and strip-plot designs have been well-documented in the literature. For instance, Goos et al. (2006) discuss the analysis of data from split-plot designs, while Jones and Nachtsheim 
(2009) review the literature on the design of split-plot experiments. An instructive article on strip-plot designs was written by Box and Jones (1992), who pointed out that robust product experiments are often performed using split-plot and strip-plot designs and contrasted the corresponding analyses. Goos and Jones (2011) discussed split-plot and strip-plot designs in much detail as well.

The weakness of split-plot MPV designs is that they provide little information concerning the whole-plot factors, resulting in large standard errors and small degrees of freedom. Therefore, hypothesis tests for the effects of these factors have poor powers and the corresponding confidence intervals are wide. The opposite is true for the sub-plot factors and for interactions between a whole-plot factor and a sub-plot factor. So, the strength of splitplot designs is that they provide much information concerning the sub-plot factors and the whole-plot-by-sub-plot interactions (i.e., the interactions between the mixture ingredients and the process variables). Another strength is that, logistically speaking, split-plot MPV designs are more convenient than completely randomized MPV designs.

Strip-plot MPV designs have two strengths and two weaknesses. One strength is that they offer much information, and thus small standard errors, many degrees of freedom, large powers and narrow confidence intervals, for the interactions between the mixture ingredients and the process variables. Another strength is that, logistically speaking, they are even more convenient than split-plot designs. The two weaknesses of strip-plot MPV designs are that the designs lead to large standard errors and few degrees of freedom for the effects of the mixture ingredients and to large standard errors and few degrees of freedom for the effects of the process variables.

The main drawback of a completely randomized MPV design is that, from a logistical viewpoint, it is the most labor-intensive experimental plan. One strength of a completely randomized MPV design is that there are no differences in degrees of freedom for the effects in the MPV model. Hence, from this perspective, all effects of interest receive an equal amount of attention. Another strength is that it allows for the simplest possible kind of regression analysis, i.e., an ordinary least squares analysis. Indeed, all other design options necessitate generalized least squares regression in combination with restricted maximum likelihood estimation for the variance components. Especially for split-plot experiments with few whole plots and strip-plot experiments with few groups and/or sets, there are too few degrees of freedom for a precise variance component estimation, and negative or zero estimates 
(depending on the default settings in statistical software) are obtained for the variance components (Goos et al., 2006). As explained by Gilmour and Goos (2009), this should not be interpreted as evidence that the variance components are truly zero, but as an indication that the experimental design does not contain sufficient information for a reliable variance component estimation. Gilmour and Goos (2009) recommend a Bayesian analysis in the event negative or zero variance component estimates are obtained.

As a final note, it is worth mentioning that the statistical weaknesses of split-plot and strip-plot designs (i.e., small degrees of freedom and large standard errors for certain effects, as well as poor variance component estimates) can be overcome to some extent by using split-plot designs with many whole plots or strip-plot designs with many groups and sets. So, some logistical convenience should be sacrificed in order to overcome the statistical weaknesses of the designs. This leads me to reiterate my advice that, for a given budget, a given availability of time and given resources, an experimenter should construct the largest possible completely randomized, split-plot and strip-plot designs feasible (taking into account logistical considerations), and evaluate these designs for given anticipated values of the variance components.

\section{References}

Akkermans, W. G., Coppenolle, H., and Goos, P. (2017). Optimal design of experiments for excipient compatibility studies. Chemometrics and Intelligent Laboratory Systems, 171:125-139.

Box, G. E. P. and Jones, S. P. (1992). Split-plot designs for robust product experimentation. Journal of Applied Statistics, 19:3-26.

Cho, T.-Y., Montgomery, D. C., and Borror, C. M. (2012). A case study involving mixture-process variable experiments within a split-plot structure. Quality Engineering, 24:80-93.

Christaki, M., Verboven, P., Van Dyck, T., Nicolaï, B., Goos, P., and Claes, J. (2017). The predictive power of batter rheological properties on cake quality - the effect of pregelatinized flour, leavening acid type and mixing time. Journal of Cereal Science, 77:219-227.

Cornell, J. A. (1981). Experiments with Mixtures: Designs, Models, and the Analysis of Mixture Data, 1st edition. New York: Wiley. 
Cornell, J. A. (1988). Analyzing data from mixture experiments containing process variables: a split-plot approach. Journal of Quality Technology, $20: 2-23$.

Cornell, J. A. (2002). Experiments with Mixtures: Designs, Models, and the Analysis of Mixture Data, 3rd edition. New York: Wiley.

Cornell, J. A. and Gorman, J. W. (1984). Fractional design plans for process variables in mixture experiments. Journal of Quality Technology, 16:20-38.

Gilmour, S. G. and Goos, P. (2009). Bayesian analysis of data from multistratum response surface designs. Journal of the Royal Statistical Society. Series C, 58:467-484.

Goos, P. and Donev, A. N. (2007). Tailor-made split-plot designs with mixture and process variables. Journal of Quality Technology, 39:326-339.

Goos, P. and Jones, B. (2011). Design of Experiments: A Case Study Approach. New York: Wiley.

Goos, P., Langhans, I., and Vandebroek, M. (2006). Practical inference from industrial split-plot designs. Journal of Quality Technology, 38:162-179.

Jones, B. and Nachtsheim, C. J. (2009). Split-plot designs: What, why, and how. Journal of Quality Technology, 41:340-361.

Kenward, M. G. and Roger, J. H. (1997). Small sample inference for fixed effects from restricted maximum likelihood. Biometrics, 53:983-997.

Kowalski, S. M., Cornell, J. A., and Vining, G. G. (2000). A new model and class of designs for mixture experiments with process variables. Communications in Statistics: Theory and Methods, 29:2255-2280.

Kowalski, S. M., Cornell, J. A., and Vining, G. G. (2002). Split-plot designs and estimation methods for mixture experiments with process variables. Technometrics, 44:72-79.

Letsinger, J. D., Myers, R. H., and Lentner, M. (1996). Response surface methods for bi-randomization structures. Journal of Quality Technology, 28:381-397. 
Næs, T., Færgestad, E. M., and Cornell, J. (1998). A comparison of methods for analyzing data from a three component mixture experiment in the presence of variation created by two process variables. Chemometrics and Intelligent Laboratory Systems, 41:221-235.

Smith, W. F. (2005). Experimental Design for Formulation. ASA-SIAM Series on Statistics and Applied Probability. Society for Industrial and Applied Mathematics. 